\title{
CONCEPTOS CLAVE QUE EXPLICAN LOS CAMBIOS EN LA PROVISIÓN DE APOYOS A LAS DISCAPACIDADES INTELECTUALES Y DEL DESARROLLO EN ESPAÑA
}

\section{Key concepts and principles that explain changes in the provision of supports for intellectual and developmental disabilities in Spain}

\author{
Miguel Ángel Verdugo Alonso \\ Universidad de Salamanca. Instituto Universitario de Integración en la Comunidad. Facultad \\ de Psicología. Avda. de la Merced, 109-131. 37005 Salamanca \\ verdugo@usal.es
}

Recepción: 3 de diciembre de 2017

Aceptación definitiva: 14 de diciembre de 2017

RESUMEN: Se presenta un análisis de los conceptos centrales que están influenciando los cambios y transformaciones en el rol de los profesionales y en el trabajo de las organizaciones de apoyo a las personas con discapacidades intelectuales y del desarrollo en España. Para ello, se abarca la necesidad de un enfoque global y sistemático de las necesidades de la persona; se resalta la importancia de la evidencia para respaldar las decisiones profesionales, de las organizaciones y de las administraciones; y se examina la influencia que los diferentes sistemas (individual, familiar, organizacional y social) tienen en la vida de la persona. Finalmente, se plantean conclusiones sobre el momento actual y el futuro inmediato.

Palabras Clave: discapacidad intelectual; discapacidad del desarrollo; apoyos; transformación organizacional; familia, sistemas; política social.

ABSTRAct: The study focuses on the analysis of the central concepts that are influencing changes and transformations in the role of professionals and in the work done 
by organizations supporting people with intellectual and developmental disabilities in Spain. This includes the need for a global and systematic approach to the needs of the person, highlighting the importance of evidence to support professional, organizations and administrations decisions, and the influence that different systems (individual, family, organizational and social) have in the life of the person. Finally, some conclusions are presented about the current moment and the immediate future.

KEY WORDs: intellectual disability; developmental disability; supports; organizational transformation; family; systems; disability policy.

\section{Introducción}

$\int$

L CAMPO DE LA DISCAPACIDAD INTELECTUAL y de las discapacidades del desarrollo estrechamente relacionadas ha evolucionado en España muy significativamente, desde mediados de los años noventa. Los cambios habidos en la concepción de discapacidad intelectual han ejercido una influencia progresiva de gran calado en las prácticas de los profesionales y de las organizaciones, cuestionando los procedimientos e inercias que eran habituales.

La evolución y la profundización de los cambios de paradigma propuestos en la concepción de discapacidad han implicado centrarse en la persona y su entorno, en la modificación de variables ambientales, frente a la consideración reduccionista de pensar exclusivamente en los "defectos” o problemas de la persona y de ahí derivar lo que hay que hacer por ella. La mejora del funcionamiento de la persona y su calidad de vida por medio de los apoyos se ha convertido en el enfoque principal de profesionales y organizaciones.

En los momentos actuales el proceso de transformación del sistema de provisión de apoyos en las organizaciones está muy presente y aceptado ampliamente, pero no de manera tan generalizada ni materializado como nos gustaría, aunque cuenta con un buen número de iniciativas que responden fielmente a las concepciones actuales. Esto nos permite ir aprendiendo, entre todos los profesionales y entre todas las organizaciones para seguir avanzando con firmeza y decisión hacia una sociedad más inclusiva que permita ejercer los derechos de las personas con discapacidad de manera similar a cualquier otro ciudadano.

A pesar de lo dicho, hay que apreciar que todavía estamos en una fase de ilusión y cambios iniciales, contando con algunas consolidaciones de esos cambios muy relevantes, pero con cierta lentitud en muchos casos. Los procesos de transformación han sido emprendidos por las organizaciones más comprometidas, mientras que otras esperan y siguen centradas en modelos anticuados, muchas veces conformistas respecto a los derechos de las personas, y muy centradas y complacidas únicamente con una situación económica más o menos estable. Es el momento en el que hemos de pasar del abuso del simple uso de la palabra "personas", tanto en las organizaciones como en la administración, para convertir a las personas con discapacidad y sus familiares en el eje central de las actividades. En la medida en que la incentivación a los proyectos e iniciativas vaya paralela, como debe, a los valores promovidos por la Convención de la ONU, habremos dado un gran salto adelante. 
A continuación, se presenta un análisis de algunos de los conceptos centrales que están influenciando los cambios y transformación en el rol de los profesionales y en el trabajo de las organizaciones de apoyo a las personas con discapacidades intelectuales y del desarrollo en España. En las distintas secciones, se abarca la necesidad de un enfoque global y sistemático de las necesidades de la persona; la importancia de la evidencia para respaldar las decisiones profesionales, de las organizaciones y de las administraciones; y la influencia que los diferentes sistemas (individual, familiar, organizacional y social) tienen en la vida de la persona. Finalmente, se plantean unas conclusiones sobre el momento actual y el futuro inmediato.

\section{La necesidad de un enfoque global y sistemático de la discapacidad}

El énfasis de la atención a la discapacidad intelectual y discapacidades de tipo cognitivo en España ha ido evolucionando desde enfoques centrados solo en lo académico o en lo laboral hacia centrarse más en la vida general de la persona. Ha sido habitual pensar en la educación solamente desde un punto de vista académico, y entender la vida adulta con un enfoque centrado exclusivamente en lo laboral. Los reduccionismos en la visión de lo que es importante para las personas se produjeron, posiblemente, por la falta de madurez de los enfoques, muchas veces dependientes de perspectivas (escuelas) educativas, psicológicas, sociales o biomédicas limitadas a considerar solo una parte de la totalidad de la vida de la persona. Pero la educación no es toda la vida y lo académico no es toda la educación. De la misma manera, el trabajo no es toda la vida y la productividad no es lo único importante en el trabajo.

La vulnerabilidad de las personas que presentan limitaciones significativas en su funcionamiento intelectual y adaptativo suele presentarse no en un área vital aislada, sino que más bien afecta a un conjunto de competencias de la persona que influyen en muchas de las áreas de su vida. Esta es la principal razón para postular ahora un enfoque holístico y sistemático. Si bien ayudar a las personas ha sido el motivo inicial común de la dedicación de los profesionales y del desarrollo de las organizaciones de apoyo, se ha tardado tiempo en saber enfocar con una visión global las necesidades de la persona más allá de las parcializaciones que los progresos del conocimiento y la experiencia iban generando en la atención individual.

No basta con analizar las diferentes áreas o partes por separado, de acuerdo a la particular cultura científica de cada profesión. Lo que un enfoque holístico significa es tener en cuenta un análisis del todo como un sistema más complejo que la suma de sus partes. Este es el esfuerzo que se debe hacer para entender bien las necesidades personales en cada momento, y así poder dar prioridad a lo que es más relevante. Para ello, se ha propuesto un enfoque multidimensional como definición constitutiva de la discapacidad intelectual (Schalock et al., 2011), y un enfoque de calidad de vida para guiar las prácticas profesionales de evaluación e intervención, todo ello con un énfasis en los apoyos para mejorar el funcionamiento individual (Schalock y Verdugo, 2002, 2007, 2012). Y, afortunadamente, esto es lo que, en España, en los tiempos actuales, mayoritariamente se ha aceptado y se sigue. 
Algunos tipos de discapacidad, probablemente por su mayor complejidad y dependencia histórica de modelos biomédicos o neurológicos, como es el caso de la parálisis cerebral, trastorno dual, autismo y otros, están encontrando más dificultades en modificar sus prácticas, el rol que desempeñan sus profesionales y la estrategia de sus organizaciones. También algunas organizaciones (públicas y privadas) que desempeñaron un papel relevante en la atención a las personas con discapacidad intelectual hace años han visto que sus modelos se quedaban anticuados, y las infraestructuras generadas a lo largo de décadas han dejado de servir apropiadamente al fin para el que se construyeron. Sin embargo, el camino a recorrer por todos es el mismo: la inclusión y participación de la persona en los ambientes naturales a los que pertenece. Hoy no se pueden justificar “jaulas de oro" o "peceras" cerradas para ninguna persona respecto a la sociedad en la que vive, independientemente de la extrema gravedad que pueda tener su discapacidad, pues estamos en un tiempo de énfasis en los derechos de la persona a participar en la comunidad, como bien establece la Convención de la ONU de 2006 (Navas, Gómez, Verdugo y Schalock, 2012; Verdugo, Navas, Gómez y Schalock, 2012).

Hoy miramos al futuro inmediato con un marco de referencia holístico, como recientemente se ha propuesto (Schalock, Luckasson, Tassé y Verdugo, en prensa), con un enfoque integrado de las cuatro principales perspectivas del conocimiento científico que nos ayudan a comprender las dificultades de las personas y a generar las decisiones prácticas oportunas para mejorar su funcionamiento y bienestar personal. Estos enfoques, cuyas características diferenciales se pueden apreciar en detalle en la Tabla 1, son: el biomédico, el psicoeducativo, sociocultural y de la justicia.

TABLA 1. Características principales de las distintas perspectivas en la discapacidad intelectual (Schalock et al., en prensa)

\begin{tabular}{|c|c|c|c|c|c|}
\hline Perspectiva & \begin{tabular}{|l} 
Principales conceptos \\
utilizados
\end{tabular} & $\begin{array}{l}\text { Supuesto "locus" } \\
\text { de la discapacidad }\end{array}$ & $\begin{array}{l}\text { Factores de riesgo } \\
\text { identificados }\end{array}$ & $\begin{array}{l}\text { Intervenciones y apoyos } \\
\text { relacionados }\end{array}$ & $\begin{array}{l}\text { Elementos típicos } \\
\text { de clasificación }\end{array}$ \\
\hline Psicoeducativa & $\begin{array}{l}\text { Aprendizaje } \\
\text { Comportamiento } \\
\text { adaptativo } \\
\text { Funcionamiento } \\
\text { intelectual } \\
\text { Motivación }\end{array}$ & $\begin{array}{l}\text { Interacción reciproca y } \\
\text { dinámica entre } \\
\text { capacidad intelectual, } \\
\text { comportamiento } \\
\text { adaptativo y participación }\end{array}$ & $\begin{array}{l}\text { Crianza } \\
\text { Ausencia de intervención } \\
\text { temprana } \\
\text { Falta de oportunidades de } \\
\text { desarrollo y crecimiento } \\
\text { personales }\end{array}$ & $\begin{array}{l}\text { Habilidades de crianza } \\
\text { Estrategias de desarrollo personal } \\
\text { Asesoramiento } \\
\text { Educación especial } \\
\text { Apoyos en la toma de decisiones } \\
\text { Tecnología de ayudas y de la } \\
\text { información }\end{array}$ & $\begin{array}{l}\text { Niveles de comportamiento adaptativo } \\
\text { Niveles de intervalos de } \mathrm{Cl}\end{array}$ \\
\hline Justicia & $\begin{array}{l}\text { Discriminación } \\
\text { Derechos legales } \\
\text { Derechos humanos }\end{array}$ & $\begin{array}{l}\text { Ordenación de la sociedad } \\
\text { Estructuras } \\
\text { gubernamentales }\end{array}$ & $\begin{array}{l}\text { Desigualdad social } \\
\text { Injusticia } \\
\text { Discriminación } \\
\text { Negación de derechos }\end{array}$ & $\begin{array}{l}\text { Afirmación de derechos } \\
\text { Planificación centrada en la persona } \\
\text { Decisiones judiciales }\end{array}$ & $\begin{array}{l}\text { Designación de: } \\
\text { competencia/incompetencia, } \\
\text { apto/no apto para los privilegios de ser } \\
\text { ciudadano }\end{array}$ \\
\hline
\end{tabular}

(C) Ediciones Universidad de Salamanca / CC BY-NC-ND

Siglo Cero, vol. 49 (1), n. ${ }^{\circ}$ 265, 2018, enero-marzo, pp. 35-52 


\section{El valor de la evidencia}

Hay ocasiones en que se justifica lo que se hace por la confianza en la inercia y rutina habitual ("porque siempre se ha hecho así... y funciona”), careciendo de reflexión autocrítica sobre las actividades desarrolladas. Se puede entender que hay dos caminos a la hora de justificar y avalar las actividades prácticas de los profesionales y de las organizaciones de apoyo: el principio de autoridad, que apela principalmente a la importancia atribuida subjetivamente a un autor o a una propuesta y no a las pruebas de los argumentos que esgrime, y el método de aportar evidencias, pruebas, que permiten basarse en hechos contrastados.

Una vez que se establece qué es lo que se debe hacer porque se conoce hacia dónde hay que dirigirse, basado en principios, valores y teorías explicativas, corresponde avanzar en definir cuál es la mejor manera de conseguir lo que se persigue y planificar cómo averiguar si se ha conseguido. Por eso, una vez que alguien nos cuenta lo que hace, hemos de preguntar cómo lo hace, cómo lo mide y qué datos tiene de ello. Sin especificar cómo lo mides y examinar los datos de resultados que obtienes, difícilmente puedes sacar conclusiones sobre el valor o la eficacia de lo que pones en marcha.

La evaluación, la intervención y la provisión de apoyos en la discapacidad intelectual y las discapacidades del desarrollo (autismo, parálisis cerebral, y otras) deben estar basadas en la evidencia, que significa combinar el conocimiento científico con la experiencia y el consenso profesional, todo ello desde un enfoque centrado en el ejercicio de los derechos de las personas.

Hay una larga tradición en la historia de la educación especial internacional y española y en el ámbito de la rehabilitación, en la cual las decisiones sobre qué estrategias educativas o rehabilitadoras eran las más apropiadas y se implementaban dependía no tanto del conocimiento científico como de la moda (siempre pasajera) de algún tipo de método o técnica con validez aparente. Se juntaba la situación de apremio percibido para dar respuestas inmediatas, para tomar decisiones "terapéuticas" ante los problemas presentados por las personas, con la incertidumbre o la ignorancia sobre cuál era lo mejor. Y la inseguridad llevaba a confiar ciegamente en estrategias o métodos que podían ser de gran utilidad para algún aspecto concreto (educativo, de apoyo al desarrollo, para mejorar la interacción social, etc.), pero no eran la panacea para afrontar todas las dificultades de las personas. Ha faltado la visión holística de la persona y ha sobrado la confianza en propuestas sin respaldo científico, que, en el mejor de los casos, son válidas solamente para algunos aspectos del funcionamiento de la persona, y en otros han respondido a intereses espurios (económicos, afán de protagonismo, enfoques disciplinares sesgados).

En los momentos actuales, por su presencia más allá de lo razonable en España y en muchos otros países, hay que denunciar lo que se conoce como psendociencia. Es común que cada cierto periodo de tiempo algunos confíen ingenuamente en una "buena nueva” tan prometedora que creen que resolverá, curará o paliará definitivamente la discapacidad intelectual y otras limitaciones significativas de la persona. Un ejemplo reciente se ha visto con la propuesta, y no olvidemos el negocio paralelo, del 
uso del té verde para mejorar el funcionamiento cognitivo de las personas con síndrome de Down. Tras cierta confusión algo generalizada para algunos profesionales y organizaciones, un artículo de reciente publicación (Stagni et al., 2017), que en España ha sido difundido por la Fundación Iberoamericana Down21 con comentarios muy oportunos, aclara la falta de evidencia del producto para la mejora del funcionamiento cognitivo. Pero hay muchos más ejemplos de procedimientos sin fundamento científico en el ámbito de la discapacidad que se trasmiten o "venden" como mágicos, sobre todo en el mundo del autismo, los trastornos generalizados del desarrollo y otras discapacidades muy significativas (p. e., magnetoterapia, homeopatía, método Padovan, método Foltra, Doman-Delacato, equinoterapia, hidroterapia, etc.). Esos procedimientos, generalmente, no son dañinos para la persona, pero suelen prometer algo parecido a la imposible curación, y sí que confunden y perjudican las expectativas de los familiares, orientando hacia prácticas injustificadas que son muchas veces económicamente gravosas y, además, adolecen de falta de fundamento científico. Eso sí, para curarse en salud, suelen recomendar simultáneamente la aplicación masiva o intensiva de tratamientos convencionales (fisioterapia, estimulación verbal, etc.) para garantizar el éxito, de tal manera que no se puede discriminar bien si la causa de éxito es el método "vendido" o el tratamiento tradicional intensivo.

Los profesionales de las comunidades médicas, educativas y del ámbito del apoyo y rehabilitación comienzan, afortunadamente, a tener presión para justificar la evidencia científica de los tratamientos que ponen en marcha y la obtención de resultados personales relevantes. El peligro está en falsear como conocimiento científico (pseudociencia) el desarrollo de prácticas, afirmaciones o creencias que no tienen resultados demostrables. Esto suele ir aparejado con una falta de teoría seria que lo sustente y con la falta de evaluaciones externas. No cabe duda de que un enfoque integral de la persona desde las cuatro perspectivas comentadas anteriormente (biomédica, psicoeducativa, sociocultural y de la justicia) es necesario, pero conviene como norma ser escéptico frente a los procedimientos “milagrosos” o “mágicos” desde cualquier enfoque. Las decisiones a tomar deben estar basadas en evidencias.

Los posicionamientos de algunas de las principales organizaciones proveedoras de apoyos y servicios en España (Plena Inclusión, Confederación Autismo) o asociaciones de profesionales representativas (AETAPI) han sido contundentes en la defensa de un enfoque científico para desenmascarar prácticas inapropiadas que en ocasiones encuentran audiencia en los medios de comunicación y revistas de divulgación profesional y confunden a las familias y los profesionales.

En la actualidad, uno de los signos del consenso positivo existente en las organizaciones españolas, sobre principios y metas, es la inclusión explícita en muchas de ellas, como misión, el centrarse en la mejora de la calidad de vida de las personas y de sus familiares. Veamos algunos ejemplos:

- Plena Inclusión: La Misión de Plena inclusión es contribuir, desde su compromiso ético, con apoyos y oportunidades, a que cada persona con discapacidad intelectual o del desarrollo y su familia puedan desarrollar su proyecto de calidad de vida, así como a promover su inclusión como ciudadana de pleno derecho en una sociedad justa y solidaria. 
- ASPACE Parálisis Cerebral: Mejorar la calidad de vida de las personas con parálisis cerebral mediante la defensa de sus derechos, los servicios a las entidades asociadas y la cooperación institucional.

- Salud Mental España: Nuestra misión es mejorar la calidad de vida de las personas con problemas de salud mental y sus familias, defender sus derechos y representar al movimiento asociativo.

- Confederación Autismo España: Su misión es apoyar a las personas con TEA y sus familias promoviendo la reivindicación y el ejercicio efectivo de sus derechos, con el propósito de favorecer su calidad de vida y conseguir la igualdad de oportunidades.

Todas las confederaciones de organizaciones, incluidas aquellas que no hacen explícita la misión de mejorar la calidad de vida, están alineadas con el concepto y proponen también el desarrollo de un enfoque de apoyos y defensa de los derechos de la persona para generar proyectos de vida individuales y con el máximo nivel de autonomía. Las palabras clave identificadoras del buen hacer en la discapacidad, en resumen, serían: Derechos, Apoyos y Calidad de vida. Otra historia es cómo en la práctica se van transformando hacia ese enfoque las organizaciones y los servicios.

\section{Las prácticas basadas en la evidencia}

En la transformación de las organizaciones, el desarrollo de prácticas basadas en la evidencia es una de las principales estrategias propuestas por Schalock y Verdugo (2012) para mejorar la provisión de apoyos individuales. Otras de las estrategias propuestas con ese fin son la apuesta por generar equipos de alto rendimiento y el desarrollo de un sistema de apoyos en las organizaciones. La mejora en competencias (formación y reciclaje) y en coordinación interna de los profesionales de los equipos, así como el desarrollo de una cultura de aprendizaje en las propias organizaciones, es una variable determinante de unas prácticas profesionales mejores y más innovadoras. El conocimiento del sistema de apoyos del que se dispone en la organización es necesario para desarrollar apropiadamente planes individuales de apoyo, así como para planificar los avances y generar estrategias de colaboración con otras entidades.

La irrupción de la preocupación por la calidad en los servicios sociales, que es característica de la llamada sociedad del bienestar, en la cual la inversión pública crece significativamente, es la causa de la preocupación por la mejora en las prácticas profesionales, la cual lleva ya un par de décadas en el ámbito de la discapacidad intelectual y del desarrollo en España. Primero, se comenzó a hablar de "buenas prácticas" y luego se han ido utilizando otros términos más o menos afortunados para identificar el "buen hacer" que sirva de modelo a otros. Este movimiento es sin duda positivo, pero conviene establecer con claridad la diferencia entre buenas prácticas y prácticas basadas en la evidencia.

Las buenas prácticas y las prácticas basadas en la evidencia comparten el que tienen como finalidad mejorar los resultados personales y de las organizaciones (Schalock, 
Verdugo y Gómez, 2011; Schalock, Gómez, Verdugo y Claes, 2017; van Loon, Bonham, Peterson, Schalock, Claes y Decramer, 2013). Las primeras se han de basar tanto en el conocimiento científico, como en los valores y estándares profesionales, y en el juicio clínico. Y las prácticas basadas en la evidencia se diferencian de ellas porque se comprometen con un nivel más alto de demostración, pues se basan en indicadores de resultados y la evaluación basada en la evidencia. Su principal utilidad es la de proporcionar la mejor evidencia disponible para tomar decisiones clínicas y organizacionales.

El conocimiento científico avanza lentamente, pero tiene la ventaja de ser acumulativo, con lo cual las certezas que aporta son progresivamente mayores. En el campo de la discapacidad, el conocimiento científico se ve "auditado" por sus implicaciones prácticas, y su valor se examina en la medida que se obtienen resultados positivos en las personas. Afortunadamente, los enfoques predominantes han cambiado sustancialmente en los últimos años, descartando rutinas y planteamientos sin una teoría y principios de base, y cuestionando las metas perseguidas. No obstante, sigue siendo necesario reclamar el desarrollo de una cultura que utilice los datos y las evidencias

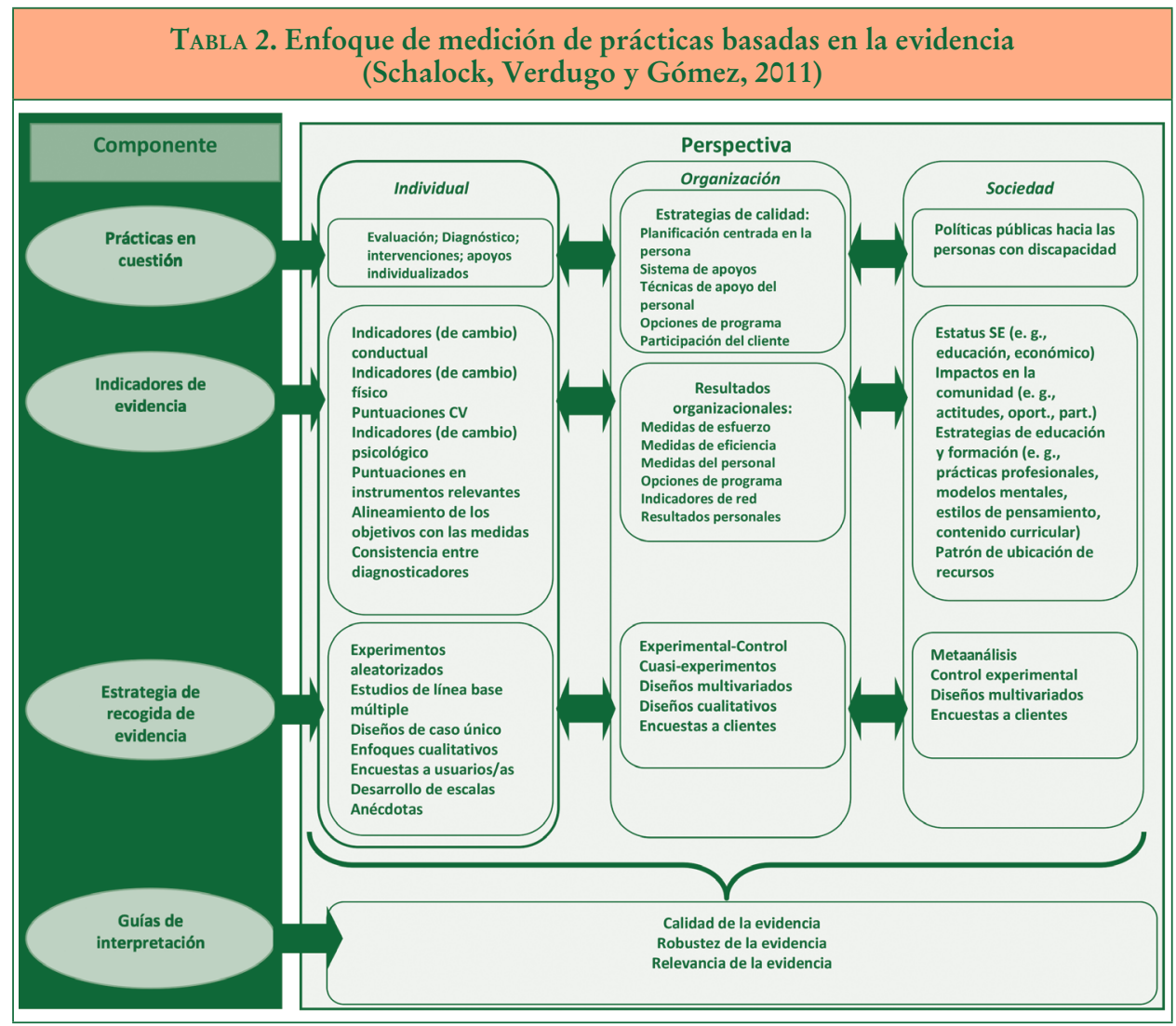

(c) Ediciones Universidad de Salamanca / CC BY-NC-ND

Siglo Cero, vol. 49 (1), n. ${ }^{\circ}$ 265, 2018, enero-marzo, pp. 35-52 
como un elemento esencial para tomar decisiones, ya sea en el ámbito clínico, organizacional o de las políticas sociales. Y esto es una tarea básica en los programas de formación de los profesionales.

Las prácticas basadas en la evidencia, desde un enfoque de calidad de vida, son aquellas que vienen avaladas por estudios de calidad que utilizan diseños rigurosos que permiten inferir y demostrar efectos significativos en resultados personales. Un resumen de un enfoque de medida de las prácticas profesionales basadas en la evidencia es el presentado en la Tabla 2. En el enfoque sistémico propuesto por Schalock et al. (2011) se exponen los diferentes tipos de prácticas (individuales, organizacionales o sociales), con los indicadores más relevantes de ellas y con las estrategias de recogida de evidencia más recomendables. La interpretación de los resultados de las prácticas se establece en base al uso de tres directrices clave: calidad, robustez y relevancia de la evidencia.

\section{La utilización de puntuaciones de calidad de vida}

Una vez que se comparte el concepto, modelo o teoría de calidad de vida individual (Schalock y Verdugo, 2002, 2012; Schalock, Verdugo, Gómez y Reinders, 2016; Van Hecke et al., 2017), sumado a la asunción de un enfoque inclusivo mediante un paradigma de apoyos, las organizaciones y sus profesionales generan importantes estrategias de cambio e innovación con la importante característica inicial común de evaluar la calidad de vida individual y la necesidad de apoyos de los usuarios. Al menos, esta es la experiencia española, se comienza evaluando y posteriormente se inicia un proceso lento de transformación de las prácticas.

La utilización de puntuaciones de calidad de vida obtenidas en evaluaciones individuales tiene como finalidad monitorizar e informar; implementar estrategias de mejora de la calidad, y establecer prácticas basadas en la evidencia para mejorar el bienestar personal (Schalock, Baker et al., en prensa).

Las puntuaciones de calidad de vida deben basarse en la aplicación de escalas de evaluación con propiedades psicométricas adecuadas (p. e., San Martín, Inico-Feaps, Gencat, Cavidace, Kidslife, Arc-Inico, etc.) y que responden a un modelo conceptual validado. Lo que se entiende por una vida de calidad es el resultado global de medir, a través de indicadores específicos en cada dominio, las ocho dimensiones del modelo de calidad de vida (Schalock y Verdugo, 2002, 2012; Schalock, Luckasson et al., en prensa): bienestar emocional, bienestar físico, bienestar material, relaciones interpersonales, inclusión social, autodeterminación, derechos y desarrollo personal.

Los indicadores específicos de calidad de vida referidos a cada dimensión o área vital son percepciones, comportamientos y condiciones que dan una indicación del bienestar de la persona. Estos indicadores están representados en los ítems de los instrumentos de evaluación de calidad de vida, y se miden habitualmente por medio de una escala de calificación tipo Likert. El proceso de construcción de una escala es el que acredita la credibilidad y aceptación de los indicadores, así como de las puntuaciones obtenidas en las subescalas y en la totalidad de las pruebas. 
La adecuación e importancia de los indicadores de calidad de vida respecto a los dominios o dimensiones de calidad de vida incluidos en un instrumento se ha de basar, en primer lugar, en un análisis riguroso de la literatura científica existente sobre la población objeto de examen. Y a partir de los resultados obtenidos, se debe enfocar un proceso cualitativo de búsqueda de consenso entre expertos reconocidos y con experiencia dilatada en las personas a las que se pretende evaluar. Para ello, en el desarrollo de escalas de evaluación en el INICO algunos autores nos basamos en una rigurosa aplicación del método Delphi, con un panel de expertos que progresivamente va logrando refinar los mejores indicadores presentados de la manera más sencilla y comprensible (Fernández, Verdugo, Gómez, Aguayo y Arias, 2017; Verdugo, Gómez, Arias, Navas y Schalock, 2014; Verdugo et al., 2013).

La fiabilidad y validez de los ítems, y de los instrumentos en sí, debe basarse, posteriormente al estudio cualitativo, en aplicaciones experimentales que permiten examinar con procedimientos estadísticos complejos el funcionamiento de la escala. Y una vez que tenemos el instrumento finalizado corresponde aplicarlo de acuerdo con la finalidad que hayamos decidido. Necesitamos un buen instrumento, pero tener un buen instrumento no garantiza que se aplique adecuadamente, al igual que ocurre con cualquier herramienta. Por eso, es importante hablar del uso de los datos o puntuaciones de calidad de vida.

Las puntuaciones en calidad de vida son relativas y no absolutas. No existe un baremo universal de calidad de vida contra el cual contrastar las puntuaciones. Por eso, es importante el desarrollo de instrumentos adaptados a la población que se va a evaluar y con muestras lo más amplias y representativas posible. De esta manera contaremos con un baremo representativo de población similar al individuo evaluado. Pero el valor de las puntuaciones siempre es relativo y, sobre todo, nos sirve para desarrollar planes individuales de mejora. Una vez que tenemos los resultados, los equipos interdisciplinares, junto a la propia persona y familia cuando proceda, deben examinarlos para extraer la información necesaria que permita planificar objetivos individuales de mejora de calidad de vida en base a estrategias de apoyo. Y también, los profesionales pueden examinar los resultados agrupados de todos los miembros de un grupo o unidad para obtener conclusiones que permitan modificar positivamente las acciones o programas habituales.

Las decisiones clínicas individuales y las decisiones organizacionales requieren sintetizar apropiadamente los resultados de las evaluaciones para, teniendo en cuenta diferentes aspectos (el individuo, la organización y el contexto), establecer prioridades y ajustar estrategias de apoyo que permitan conseguir los objetivos establecidos. Una recomendación clara sobre cómo orientar las prioridades es utilizar la planificación centrada en la persona. Las prioridades siempre deben venir avaladas por una evaluación previa de las necesidades de apoyo y un conocimiento detenido de la calidad de vida individual. La información sintetizada ha de integrarse y alinearse con un marco de resultados personales en un plan significativo para cada persona. 


\section{Distintos niveles del sistema determinan la calidad de vida}

Que la calidad de la atención y sus resultados sobre la persona dependen de las tareas realizadas en diferentes planos del sistema es algo que ha ido adquiriendo mayor importancia en los últimos años. La responsabilidad no es solamente del profesional, la familia y las organizaciones de apoyo, y el fundamento de lo que se debe realizar ahora está claro que es un derecho y no a causa de la beneficencia, caridad o bondad de las personas.

Los avances de las últimas décadas han sido muy positivos en España en distintos escenarios y niveles del sistema, pero existen todavía aspectos no resueltos o que necesitan abordarse con valentía.

\subsection{La familia y los profesionales}

En el ámbito del microsistema, en la sociedad española, se pueden apreciar en las últimas décadas, por un lado, los destacados avances en competencia y especialización de muchos profesionales y, por otro lado, el avanzado nivel organizacional y de representación política alcanzado por las organizaciones de apoyo desarrolladas por los familiares (siempre con el apoyo de profesionales comprometidos). Sin embargo, el camino solamente ha comenzado, pues las familias deben adquirir un mayor protagonismo en la vida de sus hijos, y los profesionales han de avanzar hacia un rol más comunitario incrementando sus competencias y colaboración interdisciplinar.

Los profesionales se han beneficiado en las últimas tres décadas de oportunidades de formación de postgrado y reciclaje antes inexistente en medios universitarios (p. e., los tres másteres actuales simultáneos sobre Inclusión y Calidad de Vida desarrollados por el INICO en la Universidad de Salamanca, que se iniciaron en el año 1991). Estas posibilidades de formación y actualización de conocimientos han permitido conocer los modelos más actuales e innovadores, junto a las experiencias más relevantes en un ámbito nacional e internacional, centrándose en la solución de las dificultades que tienen las organizaciones de apoyo. A su vez, las organizaciones de apoyo han promovido intensa y extensamente el aprendizaje de técnicas y estrategias de evaluación e intervención provenientes de la investigación aplicada y de la experiencia profesional acreditada (Lacasta, 2015; Tamarit, 2015). Un ejemplo claro de la formación recibida es la progresiva aplicación de los apoyos desde un enfoque de planificación centrada en la persona. Esto ha redundado en unas mejores prácticas profesionales con unos mejores resultados en las personas con discapacidad intelectual.

Un reto pendiente es la transformación del rol profesional tradicional hacia un enfoque más vinculado a la inclusión en la comunidad y menos a las respuestas exclusivamente centradas en la persona dentro del propio centro. En el mismo sentido, se ha de trasladar el centro de atención desde el servicio o centro hacia la persona y la familia, que son la misión prioritaria de las organizaciones. Hoy se entiende que los profesionales deben apoyar a las personas con discapacidad intelectual desarrollando nuevas oportunidades en su medio natural, construyendo relaciones y redes en su entorno para facilitar la inclusión y un plan de vida significativo con objetivos 
renovables constantemente, para lo cual es esencial transmitir valores inclusivos en el entorno de la persona.

Si hablamos de valores personales nos estamos refiriendo a las creencias profundas que determinan nuestra manera de ser y generan actitudes y comportamientos hacia los demás. Y en el ámbito laboral, los valores profesionales y sociales se identifican con principios y reglas de conducta positivos que representan una ética individual aceptable hacia las personas. Los valores son los que deben determinar tanto las buenas prácticas de los profesionales y de las organizaciones, como de la política pública (Verdugo, 2011). Y de ellos se han de derivar los objetivos estratégicos. Desde una perspectiva individual, los valores de dignidad, respeto, igualdad, empoderamiento, autodeterminación, no discriminación e inclusión son los que deben establecer los criterios para una buena práctica (Schalock, Baker et al., 2017). Y en ellos hay que educar a todo el entorno de la persona, en el cual desempeñan un papel primordial la familia y los profesionales, a quienes les corresponde ejemplificar el ejercicio derivado de los valores a otras personas próximas.

Una tarea pendiente para los profesionales es enfocar con criterios provenientes de teorías y del conocimiento científico la promoción de prácticas basadas en evidencias, así como consolidar y transmitir a otros los avances que se van realizando. El intercambio de experiencias y el aprendizaje de otros profesionales y de otras organizaciones juega un papel determinante para innovar y avanzar en el cambio social que requiere una sociedad inclusiva. Para ello, un reto a superar es incrementar la transmisión del conocimiento proveniente de la experiencia por medio de escribir y comunicar mejor lo que se hace. Esto es algo indispensable para que haya una fundamentación real de lo que hacemos desde una perspectiva de reflexión autocrítica.

Los familiares han ido ampliando en los últimos años su participación más allá del tradicional papel esencial y permanente de la madre hasta la activación eficaz de padres y hermanos. Los familiares han desempeñado una importante labor en el desarrollo y consolidación de las organizaciones de apoyo en España; sin embargo, han estado algo relegados de una participación más activa en los programas individuales de apoyo y en la toma de decisiones sobre aspectos esenciales para el futuro de sus hijos. En un paradigma moderno de lo que es la discapacidad entendemos que los determinantes sociales (familia, profesionales, comunidad inmediata y amplia) desempeñan un papel tan importante o más que las características personales de la discapacidad. Y, en este sentido, se hace necesario escuchar mucho más su voz, su percepción de las cosas, sus necesidades y sus prioridades de apoyo. Y esto no es solo una tarea de las organizaciones, sino que la política pública debe regular ese papel a desempeñar por la familia como garantía de los derechos de la persona y su familia (por ejemplo, en las decisiones educativas de ubicación escolar y de programas individuales de apoyo).

\subsection{La transformación de las organizaciones sociales y los servicios}

En el nivel del mesosistema, junto al extenso crecimiento de servicios y la consolidación de muchas organizaciones en los años recientes en España, se aprecia también 
una apuesta por el desarrollo de buenas prácticas y procesos importantes de transformación de los distintos tipos de servicios ofertados, desde una perspectiva de redes, impulsados con decisión desde las confederaciones y redes de las organizaciones (Lacasta, 2015; Tamarit, 2015). Un claro exponente es el proceso de transformación promovido desde Plena Inclusión España, que plantea unos servicios centrados en la persona, dando relevancia al rol de ciudadanía plena, los apoyos personalizados y las oportunidades de inclusión, a la vez que se asesoran procesos de cambio organizacional con una red de consultoría.

A pesar de lo expuesto, existen todavía resistencias importantes al avance progresivo de algunas organizaciones hacia un modelo comunitario por diferentes causas: carencia de información y formación apropiadas en el personal responsable, inseguridad y temor a los cambios, falta de incentivación financiera por parte de las administraciones, intereses personales y organizacionales, comodidad y apatía, y otros. La estrategia para superar esa situación de impasse en algunas organizaciones es potenciar un modelo de cambio basado en valores y centrado en el contexto (Verdugo, Jenaro, Calvo y Navas, 2017). Hoy, los valores y principios para implementar los cambios han de acomodarse a las directrices de la Convención de Naciones Unidas sobre los Derechos de las Personas con Discapacidad, que resaltan la no discriminación y promueven la participación e inclusión total en la sociedad.

Los valores asumidos vuelven a ocupar en este caso un papel predominante. Los valores profesionales son los que conforman la visión, misión y cultura de las organizaciones. La importancia de los valores viene establecida además porque son los que fundamentan los modelos mentales en los que se enraízan las asunciones, generalizaciones e imágenes que el personal de las organizaciones tiene para comprender y actuar (Schalock, Baker et al., 2017; Schalock y Verdugo, 2012, 2013).

La responsabilidad de las organizaciones y de sus profesionales exige el desarrollo de actitudes centradas en respetar al individuo y desarrollar atención centrada en la persona; poner el énfasis en los derechos humanos y legales, e implicarse en prácticas de apoyo que facilitan la calidad de las interacciones de la persona y facilitan su bienestar. Algunos componentes que se han destacado del rol de responsabilidad de los profesionales son (Schalock y Keith, 2016): conocer las tendencias actuales que tienen impacto en el área (convención de la ONU, concepto de calidad de vida y sus implicaciones, paradigma de apoyos, empoderamiento de las personas); estar bien entrenados en las mejores prácticas actuales (basadas en evidencias); actuar de acuerdo a un código ético; ejercer habilidades de pensamiento crítico, y apoyar al individuo con el desarrollo de nuevas oportunidades, construyendo su proyecto vital en el entorno comunitario natural.

La transformación de las organizaciones va en una dirección de mejora de la eficacia y eficiencia para poder tener una sostenibilidad apropiada en el futuro. En nuestro país ya se ha dado por muchas organizaciones un salto cualitativo, sobre todo en los servicios sociales, hacia un compromiso claro con los sistemas de calidad para mejorar el trabajo que se realiza. Por un lado, las organizaciones y, por otro lado, las administraciones (aunque con diferencias según cada comunidad autónoma) implementan sus propios sistemas de calidad basados en el modelo de excelencia de la Fundación 
Europea para la Gestión de la Calidad (EFQM) y las normas ISO (Organización Internacional de Normalización o Estandarización) como sistema de gestión de la calidad y, afortunadamente, complementados habitualmente con un enfoque de mejora de la calidad de vida individual. Sin este último los esfuerzos realizados se quedarían concentrados en los procesos burocráticos y organizacionales perdiendo el norte de la persona y su bienestar como referencia.

La sostenibilidad futura de las organizaciones debe entenderse como la capacidad de una organización para adaptarse con éxito al cambio y maximizar sus recursos para proporcionar una amplia gama de oportunidades y actividades en sus servicios que den como resultado productos organizacionales y resultados personales valiosos (Schalock, Verdugo y Lee, 2016; Schalock, Verdugo y van Loon, 2018). A su vez, el alineamiento vertical y horizontal de las iniciativas favorecerá la transformación de las organizaciones (Schalock, 2017; Verdugo et al., 2017).

Estos aspectos comentados exigen un análisis crítico por parte de las organizaciones y administraciones españolas, pues apenas hemos comenzado el camino. El modelo organizacional que se ha desarrollado en las organizaciones de servicios sociales españolas desde los años sesenta del siglo pasado ha tenido un largo y sufrido caminar hasta consolidarse y presentar un paisaje asombroso y envidiable caracterizado por la existencia de redes potentes coordinadas en sus esfuerzos. Pero este modelo no es el que nos vale para el futuro, hay que abrir las organizaciones y sus servicios hacia el exterior para situarse en los ambientes naturales de la persona (familia, educación, empleo, comunidad) y ayudar a construir una sociedad diferente. Sin una sociedad más activa la inclusión puede ser una quimera. Y aquí, el papel de la política pública también es esencial.

\subsection{La politica social de las administraciones}

Si examinamos aspectos del macrosistema, podemos destacar el progresivo papel que las administraciones estatal y autonómica han ido asumiendo en la aportación de recursos, y en la actualización de las leyes y normas reguladoras de la atención. Pero la principal contradicción se da entre el discurso que se pronuncia y las acciones que deberían realizarse. Los responsables de la política pública saben que es correcto y rentable identificarse con las personas con discapacidad y sus necesidades (y usan y abusan de la imagen todo lo que pueden), pero no actúan en consecuencia a la hora de aportar recursos y regular apropiadamente la incentivación de los cambios que se deben hacer.

Como dicen Turnbull y Stowe (2017), en la implementación de la política social una cosa son los formalismos legales, "política en los libros”, que nosotros en España podemos decir "política en las normas y regulaciones", y otra el realismo legal, "política en las calles”. Los responsables de la política pública española central y autonómica suelen comprometerse exclusivamente con un cambio en las normas y nunca con el cambio real en las calles. Para eso, dejan que sean las organizaciones y sus profesionales quienes se "batan el cobre". Y, si no hay compromiso con los cambios en la calle, hay que entender que todo se queda en florituras verbales o apariencia de compromiso. Por eso, debemos hacer obligatorio que, cuando se hacen propuestas, 
programas, regulaciones o compromisos, estos se traduzcan en indicadores concretos que permitan su evaluación.

Otra de las principales carencias de la actuación de las administraciones españolas es la despreocupación por evaluar los resultados de sus acciones. Evaluar está íntimamente ligado a la eficacia y eficiencia de las inversiones realizadas y de los objetivos establecidos. Sin evaluación no hay constatación de lo prometido. Y ha llegado el momento de que se una el análisis de lo prometido con la evaluación de lo conseguido. Nadie, en el mundo de la discapacidad, debe sentirse satisfecho con las promesas, si no vienen acompañadas de indicadores específicos que permitan comprobar lo que se promete. Esto hoy es una exigencia básica para la credibilidad de los responsables de la política pública. Se hace necesario urgentemente el desarrollo de una cultura de evaluación que contribuya a mejorar el rigor analítico de los avances en el bienestar de las personas y en su inclusión social (Rodríguez, 2015).

Ha llegado el momento de exigir un giro en la planificación estratégica general, sincronizando adecuadamente las diferentes fases del proceso: los principios de los que se parte (derechos, no discriminación, inclusión, etc.) y los resultados (indicadores) que se persiguen, la implementación estatal y autonómicas (alineando horizontal y verticalmente los niveles del sistema) y la evaluación de resultados basada en indicadores (Schalock, 2017; Shogren, Luckasson y Schalock, 2017; Verdugo, Jenaro, Calvo y Navas, 2017). Desde una perspectiva holística centrada en mejorar la calidad de vida de las personas, se puede establecer un marco de referencia que integre y alinee las metas de la política pública (dignidad humana y autonomía, esfuerzo humano satisfactorio a nivel personal y compromiso humano), las dimensiones de resultados personales (derechos humanos y legales, bienestar material, bienestar físico, bienestar emocional, autodeterminación, desarrollo personal, relaciones interpersonales e inclusión social) y los indicadores de resultados claramente precisados.

En todos los planos del sistema, la distribución de recursos y el desarrollo de oportunidades en España deben fluir en el futuro en la dirección de los cambios propuesta por Schalock en este mismo número de la revista (ver Figura 1, en Schalock, 2018). Por un lado, la administración y las organizaciones deben ir tomando decisiones dirigidas a que los recursos financieros y de otro tipo puedan ir cada vez más directamente a las familias y a las personas; $y$, por otro lado, las familias y organizaciones deben promover más oportunidades inclusivas en la sociedad. Y no nos olvidemos de la necesidad perentoria de involucrar a la empresa ordinaria y organizaciones sociales de otro tipo (sindicatos, fundaciones, asociaciones culturales, etc.) para gestionar y tejer la red natural de apoyos desde la misma sociedad, sin dar lugar solo a lo "especial" y no asumir o reclamar exclusivamente la responsabilidad de hacer las cosas. La discapacidad es un asunto de toda la sociedad y las respuestas deben estar diversificadas.

\section{Conclusiones y una mirada al futuro}

Decía el conocido e influyente psicólogo norteamericano Abraham Maslow que:

cuando cambia la filosofía del hombre (su naturaleza, sus objetivos, sus potencialidades, su realización), entonces todo cambia. No solo cambia su filosofía política, económica, 
ética y su filosofía de la historia, sino también su filosofía de la educación, de la psicoterapia y del desarrollo personal, la teoría acerca de cómo ayudar a las personas a llegar a ser aquello que pueden y necesitan profundamente llegar a ser. Nos encontramos en la actualidad en el centro de un cambio de este tipo en torno a las capacidades, potencialidades y objetivos del hombre. Está naciendo una nueva concepción acerca del hombre y de su destino, y sus implicaciones son muchas, no sólo para nuestras concepciones educativas, sino también para lo que hace a la ciencia, política, literatura, economía, religión e incluso a nuestras concepciones del mundo no-humano (1968/1972: 251).

Lo dicho hace cincuenta años por Maslow se puede trasladar con facilidad a la visión positiva actual que tenemos de las personas con discapacidad, particularmente de aquellas con discapacidades intelectuales y del desarrollo más significativas. Los derechos humanos son los mismos para todos, y el ejercicio de los mismos es una responsabilidad de toda la sociedad. Lo que propone la convención de la ONU es un mapa de ruta obligatorio no solo para cambiar normas y regulaciones, sino para transformar la sociedad. Y para transformar la sociedad hemos de comenzar por transformar las organizaciones de apoyo, empoderar más a las personas y sus familiares, mejorar nuestras prácticas profesionales y organizacionales, y dar un salto cualitativo en la planificación estratégica de la política pública.

¿Por dónde hemos de comenzar?

Lo primero es saber dónde estamos en una perspectiva evolutiva histórica de los paradigmas que han inspirado el buen hacer. $\mathrm{Y}$ es que hemos evolucionado primero desde las prácticas institucionales hacia los servicios en la comunidad hacia finales del siglo pasado, y ahora estamos caminando desde los servicios hacia los apoyos individuales en el entorno natural de cada persona.

Hay que actuar en los tres niveles descritos del sistema, y avanzar en todos ellos, persiguiendo la alineación de los esfuerzos realizados. Y lo primero es cambiar los modelos mentales del personal de atención directa, de los directivos de las organizaciones y de los responsables de la política pública. Además, hay que incentivar constantemente la innovación y el cambio para mejorar. La responsabilidad de las administraciones en este caso es de máxima relevancia.

Hay que hacer las transformaciones requeridas con prudencia y seguridad, pero sin descanso. Para ello, se necesitan organizaciones y profesionales con valentía para modificar las rutinas e inercias que puedan existir en la actividad cotidiana. Y necesitamos políticos comprometidos y también valientes para mirar directamente a los problemas y dificultades y no delegar en otros la solución. Y, finalmente, no olvidemos la necesidad de incrementar la investigación aplicada comprometida con los procesos de cambio y mejora. Esa investigación es la que, colaborando con organizaciones y administraciones, puede aportar rigor y calidad, metodología, evaluación, análisis independiente, desarrollo de herramientas, y otros aspectos necesarios en los procesos de mejora y transformación. Sin estrategias específicas de incentivación de la investigación todo queda a la libre voluntad de algunos. Y así nos va. 
CONCEPTOS CLAVE QUE EXPLICAN LOS CAMBIOS EN LA PROVISIÓN DE APOYOS

A LAS DISCAPACIDADES INTELECTUALES Y DEL DESARROLLO EN ESPAÑA

MIGUEL ÁNGEL VERDUGO ALONSO

\section{Referencias bibliográficas}

Fernández, M., Verdugo, M. Á., Gómez, L. E., Aguayo, V. y Arias, B. (2017). Core indicators to assess quality of life in population with brain injury. Social Indicators Research, 1-16. doi: 10.1007/s11205-017-1612-6.

LACASTA, J. J. (2015). FEAPS, 50 años de unión por las personas con discapacidad intelectual y por sus familias. Siglo Cero, 46 (1), 41-65.

Maslow, A. (1972). El hombre autorrealizado. Hacia una psicología del ser. Madrid: Kairós.

Navas, P., Gómez, L. E., Verdugo, M. Á. y Schalock, R. L. (2012). Derechos de las personas con discapacidad intelectual: Implicaciones de la Convención de Naciones Unidas. Siglo Cero, 43 (3), 7-28.

Rodríguez, G. (2015). Prólogo. En J. Calero, X. Fontcuberta y A. García, Manual práctico de evaluación para políticas públicas en el ámbito de la discapacidad (pp. 9-12). Madrid: CINCA, colección Cermies.

SCHALOCK. R. L. (2017). Introduction to the special issue on disability policy in a time of change. Intellectual and Developmental Disabilities, 55 (4), 215-222. doi: 10.1352/1934-955655.4.215.

Schalock, R. L. (2018). Seis ideas que están cambiando el campo de las discapacidades intelectuales y del desarrollo en todo el mundo. Siglo Cero, 49 (1), 265.

Schalock, R. L., Baker, A., Claes, C., González, J., Malatest, R., van Loon, J., Verdugo, M. Á. y Wesley, G. (en prensa). The use of quality of life scores for monitoring and reporting, quality improvement, and research. Journal of Policy and Practice in Intellectual Disabilities.

Schalock, R. L., Borthwick-Duffy, S. A., Bradley, V., Buntix, W. H. E., Coulter, M. D., Craig, E. M., ... y Yeager, M. H. (2011). Discapacidad intelectual. Definición, clasificación y sistemas de apoyos. 11. a edición. Madrid: Alianza Editorial. [Trad. M. Á. Verdugo. Original: Intellectual disability. Definition, Classification, and Systems of Supports. $11^{\text {th }}$ Edition. Washington, D.C.: American Association on Intellectual and Developmental Disabilities].

Schalock, R. L., Gómez, L. E., Verdugo, M. Á. y Claes, C. (2017). Evidence and EvidenceBased Practices: Are We There Yet? Intellectual and Developmental Disabilities, 55 (2), 112-119.

Schalock, R. L. y KeITH, K. D. (2016). Cross-cultural quality of life: Enhancing the lives of persons with disability. Washington, DC: American Association on Intellectual and Developmental Disabilities.

Schalock, R. L., Luckasson, R., Tassé, M. J. y Verdugo, M. Á. (en prensa). A holistic theoretical approach to intellectual disability: Going beyond the four current perspectives. Intellectual and Developmental Disabilities.

SCHALOCK, R. L. y VERDUGO, M. Á. (2002). Handbook on quality of life for human service practitioners. Washington, DC: American Association on Mental Retardation. [El concepto de calidad de vida en los servicios y apoyos para personas con discapacidad intelectual. Madrid: Alianza, 2003].

Schalock, R. L. y Verdugo, M. Á. (2007). El concepto de calidad de vida en los servicios y apoyos para personas con discapacidad intelectual. Siglo Cero, 38 (4), 21-36.

SCHAlOCK, R. L. y Verdugo, M. Á. (2012). A leadership guide for today's disabilities organizations: Overcoming challenges and making change happen. Baltimore: Brookes. [El cambio en las organizaciones de discapacidad. Estrategias para superar sus retos y hacerlo realidad. Guía de liderazgo. Madrid: Alianza, 2013]. 
Schalock, R. L. y Verdugo, M. Á. (2013). The transformation of disabilities organizations. Intellectual and Developmental Disabilities, 51, 273-286.

Schalock, R. L., Verdugo, M. Á. y Gómez, L. E. (2011). Evidence-based practices in the field of intellectual and developmental disabilities: An international consensus approach. Evaluation and Program Planning, 34 (3), 273-282.

Schalock, R. L., Verdugo, M. Á., Gómez, L. E. y Reinders, H. S. (2016). Moving us toward a theory of individual quality of life. American Journal on Intellectual and Developmental Disabilities, 121 (1), 1-12.

Schalock, R. L., Verdugo, M. Á. y Lee, T. (2016). A systematic approach to an organization's sustainability. Evaluation and Program Planning, 56, 56-63.

SCHALOCK, R. L., Verdugo, M. Á. y van LoOn, J. (2018). Understanding organization transformation in evaluation and program planning. Evaluation and Program Planning, 67, 53-60.

Shogren, K. A., Luckasson, R. y Schalock, R. L. (2017). An integrated approach to disability policy development, implementation, and evaluation. Intellectual and Developmental Disabilities, 55 (4), 258-268. doi: 10.1352/1934-9556-55.4.258.

Stagni, F., Giacomini, A., Emili, M., Guidi, S., Ciani, E. y Bartesaghi, R. (2017). Epigallocatechin gallate: A useful therapy for cognitive disability in Down syndrome? Neurogenesis, 4 (1), e12700383. http:/dx.doi.org/10.1080/23262133.2016.1270383. [Traducido al español en Revista Virtual Síndrome de Down, 2017, 192. http://www.down21. org/revista-virtual/1731-revista-virtual-2017/revista-virtual-sindrome-de-down-mayo2017-n-192/3065-editorial-mayo-2017.html].

TAMARIT, J. (2015). La transformación de los servicios hacia la calidad de vida. Una iniciativa de innovación social de FEAPs. Siglo Cero, 46 (3), 47-71.

Turnbull, R. y Stowe, M. (2017). A model for analyzing disability policy. Intellectual and Developmental Disabilities, 55, 223-233.

Van Hecke, N., Claes, C., Vanderplasschen, W., De Maeyer, J., De Witte, N. y VandevelDE, S. (2017). Conceptualisation and Measurement of Quality of Life Based on Schalock and Verdugo's Model: A Cross-Disciplinary Review of the Literature. Social Indicators Research, 1-17.

Van Loon, J. H., Bonham, G. S., Peterson, D. D., Schalock, R. L., Claes, C. y Decramer (2013). The use of evidence-based outcomes in systems and organizations providing services and supports to persons with intellectual disability. Evaluation and Program Planning, 36 (1), 80-87.

Verdugo, M. Á. (2011). Dignidad, igualdad, libertad, inclusión, autodeterminación y calidad de vida. Siglo Cero, 42 (240), 18-23.

Verdugo, M. Á., Gómez, L. E., Arias, B., Navas, P. y Schalock, R. L. (2014). Measuring quality of life in people with intellectual and multiple disabilities: Validation of the San Martín scale. Research in Developmental Disabilities, 35 (1), 75-86.

Verdugo, M. Á., Gómez, L. E., Arias, B., Santamaría, M., Clavero, D. y Tamarit, J. (2013). Evaluación de la calidad de vida en personas con discapacidad intelectual: la escala INICOFEAPS. Siglo Cero, 44 (3), 6-20.

Verdugo, M. Á., Jenaro, C., Calvo, I. y Navas, P. (2017). Disability policy implementation from a cross-cultural perspective. Intellectual and Developmental Disabilities, 55 (4), 234246. doi: 10.1352/1934-9556-55.4.234.

Verdugo, M. Á., Navas, P., Gómez, L. E. y Schalock, R. L. (2012). The concept of quality of life and its role in enhancing human rights in the field of intellectual disability. Journal of Intellectual Disability Research, 56, 1036-1045. 